\title{
Minimization of Fuel Cost and Emission for Thermal Units by Using an Effective Meta-heuristic Algorithm
}

\author{
Cuong Duc Minh Nguyen \\ Faculty of Electrical and Electronics Engineering, Ly Tu Trong Technical \\ College, Ho Chi Minh City, Vietnam \\ Email:duccuongltt@gmail.com
}

\begin{abstract}
The paper presents the application of improved Particle Swarm optimization algorithm (IPSO) for solving combined economic and emission load dispatch (CEED) problem where transmission power losses are considered. In CEED problem, two objective functions are included to be minimized consisting of fuel cost and emission in addition to the consideration of valve point loading effects on thermal units. The method is developed by modifying the several modifications on the conventional Particle Swarm optimization (CPSO) in aim to improve the performance of the original one. IPSO is tested on two three-unit systems with the transmission power losses but without the valve effects and one ten-unit system with the transmission power losses and the vale effects. The performance of IPSO is evaluated by comparing obtained results with the CPSO and other existing algorithms available in the study. As a result, it can be concluded that the proposed method outperforms others and is very strong for solving the CEED problem.
\end{abstract}

Keywords: Improved Particle Swarm optimization, transmission power losses, economic load dispatch, emission dispatch, combined economic and emission dispatch

\section{Introduction}

The main task of the CEED problem is to determine the optimal power output of thermal units so that the fuel cost and emission can be minimized significantly while exactly meeting all constraints from current set of units and power system such as limitations on capacity of thermal units, power balance constraints considering power losses in transmission lines. The fact that fossil fuels will become exhausted in the near future because people have exploited and used the fuels with a large amount yearly. Furthermore, during the process of generating electricity, polluted emissions such as NOx, $\mathrm{SO} 2$ and $\mathrm{CO} 2$ are released into the air without control. Consequently, the purpose of minimization of both fuel cost and emission has a significantly high role in the power systems. Due to the importance of the problem, a huge number of researchers have been attracted and published a lot of papers so far such as Hopfield Neural Network (HNN) [1], Improved Hopfield Neural Network Model (IHNN) [1], Tabu Search (TS) [2], fuzzy logic controlled genetic algorithm (FCGA) [3], the Non-dominated Sorting Genetic Algorithm - II (NSGAII) [4], Differential Evolution (DE) [5], Genetic algorithm (GA) [6], Particle swarm optimization (PSO) [6], biogeography-based optimization (BBO) [7], pareto differential evolution (PDE) [8], nondominated sorting genetic algorithm-II (NSGAII) [8], strength pareto evolutionary algorithm 2 (SPEA 2) [8], Hybrid Differential evolution-sequential quadratic programming (DE-SQP) [8], Hybrid Particle Swarm optimization- sequential quadratic programming (PSO-SQP) [9], parallel synchronous PSO algorithm (PSPSO) [10], ABC_PSO [11], multi-objective cultural algorithm (MOCA) [12], Basic Cuckoo Search Algorithm (CSA) [13], Lambda method (LM) [14], Hopfield Lagrange Network (HLN) [14], flower pollination 
algorithm (FPA) [15], Bat algorithm [16], modified Bat algorithm (MBA) [16], and gravitational search algorithm (GSA) [17]. Among these considered methods, IHNN [1], LM [14] and HNN [14] belong to the family of deterministic algorithms where other ones are included in the meta-heuristic algorithms. A big difference between the two ones is the way for optimal solution obtained. In fact, the comer owns only one solution and the solution tends to be improved gradually when the search process goes to the end whereas the latter consists of a set of solutions, which are improved by evaluating fitness function. When the search process of the comer ends, it means that all the constraints can be exactly met; however, the manner is not always true for the latter. The latter's stopping criteria is always based on the maximum number of iterations and all constraints can be exactly satisfied although the search process does not terminate. On the other hand, it can be concluded that the applicability of the meta-heuristic algorithm is more potential than the deterministic ones because the deterministic ones must stop dealing with problems where nonconvex function as well as non-differential functions are included meanwhile the meta-heuristics can solve easily. The HNN method was an efficient neural network for dealing with optimization problems. In order to solve the hydrothermal scheduling problem, HNN first constructed an energy function containing fuel cost objective function, the square of available water constraint, power balance constraint and power losses in transmission lines accompany with four different coefficients corresponding to the four terms in the energy function. The key word for dealing with all constraints and fast convergence to optimal solution is completely dependent on the four coefficients; however, the selection of the coefficients is not an easy task when applying HNN to the problem. Therefore, HNN method also suffered slow convergence to optimal solution and the constraints of the problem must be linearized when applying in HNN. Compared to HNN, IHNN is more effective since it can converge to better optimal solution faster. However, the two methods have the same drawback that they could not deal with a large scale problem easily. On the contrary, HLN can yield an optimal solution very fast and there is no impact from the large scale system but only from large number of constraints. In [16], both conventional BA and its modified version have been applied for solving the problem. Via the result comparison, it indicates that MBA is more effective than BA for one three unit system. Clearly, the evidences from one three unit system are not enough to evaluate the performance of the MBA.

Particle swarm optimization (PSO) is a population based optimal search algorithm developed by Kennedy and Eberhart in 1995 [18]. In the conventional PSO, each individual particle searches in space by adjusting its velocity based on both its own previous best location and its neighbors' best location at each time step. The effectiveness and robustness of the PSO have been improved by performing modifications [19], [20]. To validate the effectiveness and robustness of the applied method, two systems with three units and power losses in transmission lines neglecting valve point loading effects and one system with ten units considering both power losses and valve point loading effects. The comparisons among the method with others have indicated that the IPSO method is efficient for CEELD problem.

\section{Problem Formulation}

\subsection{Objective Function}

In the CEED problem, the fuel cost and polluted emission of each generating unit are minimized, thus the objective of the problem is written as: 
$\operatorname{Min} \sum_{i=1}^{N} F_{i}=\sum_{i=1}^{N}\left[w_{1} F_{1 i}\left(P_{i}\right)+w_{2} \cdot P R \cdot F_{2 i}\left(P_{i}\right)\right]$

Where $w_{1}$ and $w_{2}$ are weight factor associate with fuel cost and emission; $P R$ is the price penalty factor [21]; $F_{1 i}$ and $F_{2 i}$ are the fuel cost function and emission function of thermal unit $i$.

The fuel cost function and emission function are represented by.

$F_{1 i}\left(P_{i}\right)=a_{i}+b_{i} P_{i}+c_{i} P_{i}^{2}$

$F_{2 i}\left(P_{i}\right)=\sum_{i=1}^{N}\left(\mathrm{a}_{e i} P_{i}^{2}+b_{e i} P_{i}+c_{e i}\right)$

Where $N$ is the number of generators; $P_{i}$ is real power output of generator $i ; P_{i, \min }$ is minimum power output of unit $i ; a_{i}, b_{i}, c_{i}$ and $a_{e i}, b_{e i}, c_{e i}$ are fuel cost and emission coefficients of unit $i$, respectively.

In addition, when considering the valve point loading effects during the process of increasing or decreasing power, the fuel cost function is approximately presented by a combination of both quadratic function and sinusoidal function as follows

$F_{i}\left(P_{i}\right)=a_{i}+b_{i} P_{i}+c_{i} P_{i}^{2}+\left|e_{i} \times \sin \left(f_{i} \times\left(P_{i, \text { min }}-P_{i}\right)\right)\right|$

Furthermore, the form of the emission function is also more complicated than the quadratic curve shown in (3) and it is expressed by the combination of quadratic function and exponential function as follows

$F_{2 i}\left(P_{i}\right)=\sum_{i=1}^{N}\left[\mathrm{a}_{e i} P_{i}^{2}+b_{e i} P_{i}+c_{e i}+d_{e i} \exp \left(f_{e i} P_{i}\right)\right]$

\subsection{Constraints}

Real power balance: the total real power output of generating units satisfies total load demand plus system power losses.

$\sum_{i=1}^{N} P_{i}=P_{D}+P_{L}$

where $\mathrm{P}_{\mathrm{D}}$ is total system load demand; $\mathrm{P}_{\mathrm{L}}$ is total transmissin loss and is expressed by:

$P_{L}=\sum_{i=1}^{N} \sum_{j=1}^{N} P_{i} B_{i j} P_{j}+\sum_{i=1}^{N} B_{0 i} P_{i}+B_{00}$

Generator capacity limits: the real power output os geerating units should be limited between theris upper anh lower bounds represented by:

$P_{i \text { min }} \leq P_{i} \leq P_{i \max }$

where $P_{i, \min }$ and $P_{i, \max }$ are maximum and minimum power outputs of unit i.

\section{Improved Particle Swarm Optimization}

\subsection{Conventional PSO}

The conventional PSO was first developed by Kennedy and Eberhart [16] in 1995. Similar to other meta-heuristic algorithms, the PSO algorithm consists of $N_{p}$ particles with their position $X_{d}$ and velocity $V_{d}, d=1, \ldots, N_{p}$ where each particle $d$ contains a solution for 
the problem. The velocity of each particle $d$ is updated by using the exchange information among its current position with its best previous position and its neighbor's best previous position. The best previous position of particle $d$ and the best previous of the $d^{\text {th }}$ particle's neighbor are respectively represented by Pbest $_{d}$ and Nbest $t_{d}, \mathrm{~d}=1, \ldots, N_{p}$. The new velocity and position of the particle $d$ are updated as follows:

$V_{d}^{\text {new }}=V_{d}+c_{1} \cdot$ rand $_{1} \cdot\left(\begin{array}{l}\text { Pbest }_{d} \\ -X_{d}\end{array}\right)+c_{2} \cdot$ rand $_{2} \cdot\left(\begin{array}{l}\text { Nbest } \\ -X_{d}\end{array}\right)$

$X_{d}^{n e w}=X_{d}+V_{d}$

where $c_{1}$ and $c_{2}$ are acceleration constants; $\operatorname{rand}_{1}$ and $\operatorname{rand}_{2}$ are random numbers with uniform distribution between 0 and 1 .

\subsection{Local Vision of PSO with Constriction Factor (LCPSO)}

By using the information from the best previous position of the two other neighbor and constriction factor, the velocity of the LCPSO is updated as below:

$V_{i}^{\text {new }}=K\left(V_{i}+c_{1} \cdot c d_{1} \cdot\left(\begin{array}{l}\text { Pbest }_{d} \\ -X_{d}\end{array}\right)+c_{2} \cdot c d_{2} \cdot\left(\begin{array}{l}\text { Lbest }_{d} \\ -X_{d}\end{array}\right)\right)$

$K=0.73$

where Lbest $_{d}$ is the best one among Lbest $_{d-1}$, Lbest $_{d}$ and Lbest $_{d+1}$ of $(d-1)^{\text {th }}$ particle, $d^{\text {th }}$ particle, and $(d+1)^{\text {th }}$ particle.

\section{Implementation of IPSO for CEED Problem}

\subsection{Initialization}

A population of $N_{P}$ nests or $\mathrm{N}_{\mathrm{P}}$ particle is represented by $X_{d}\left(d=1, \ldots, N_{p}\right)$ where each solution corresponding to each egg or each particle's position given by $X_{d}=\left[P_{2, d}, P_{3, d}, \ldots, P_{N, d}\right]$. The power outputs are randomly initialized satisfying the limitation, $P_{i, \min } \leq P_{i, d} \leq P_{i, \max }$

The fitness function of each solution for the considered problem is calculated as:

$F T_{d}=\sum_{i=1}^{N}\left[w_{1} F_{1 i}\left(P_{i}\right)+w_{2} \cdot P R \cdot F_{2 i}\left(P_{i}\right)\right]+K_{s} \times\left(P_{1, d}-P_{1}^{\lim }\right)^{2}$

Where $K_{s}$ is the penalty factors; and $P_{1, d}$ is the power output of slack thermal unit $1[14]$ and obtained as follows:

$P_{1}=P_{D}+P_{L}-\sum_{i=2}^{N} P_{i}$

The power loss equation in (7) is rewritten with respect to an unknown variable of by $P_{1}$ as:

$P_{L}=B_{11} P_{1}^{2}+\left(2 \sum_{i=2}^{N} B_{1 i} P_{i}+B_{01}\right) P_{1}+\sum_{i=2}^{N} \sum_{j=2}^{N} P_{i} B_{i j} P_{j}+\sum_{i=2}^{N} B_{0 i} P_{i}+B_{00}$

Substituting $P_{L}$ in (15) into (14), a quadratic equation is obtained:

$A \times P_{1}^{2}+B \times P_{1}+C=0$

where the coefficients $A, B$ and $C$ are determined by:

$A=B_{11}$ 


$$
\begin{aligned}
& B=2 \sum_{i=2}^{N} B_{1 i} P_{i}+B_{01}-1 \\
& C=\sum_{i=2}^{N} \sum_{j=2}^{N} P_{i} B_{i j} P_{j}+\sum_{i=2}^{N} B_{0 i} P_{i}+B_{00}+P_{D}-\sum_{i=2}^{N} P_{i}
\end{aligned}
$$

The power output of the slack unit with positive value is chosen between the two roots obtained by solving second order equation (16) as below:

$P_{1}=\frac{-B \pm \sqrt{B^{2}-4 \times A \times C}}{2 A}$, where $B^{2}-4 \times A \times C \geq 0$

And the limit for slack thermal unit 1 in (13) is determined as follows:

$$
P_{1}^{\mathrm{lim}}=\left\{\begin{array}{cc}
P_{1, \max } & \text { if } P_{1, d}>P_{1, \max } \\
P_{1, \min } & \text { if } P_{1, d}<P_{1, \min } \\
P_{1, d} & \text { otherwise }
\end{array}\right.
$$

Where $P_{l, \max }$ and $P_{l, \min }$ are the maximum and minimum power outputs of slack thermal unit 1 , respectively.

\subsection{One System Considering Value Point Effects on Thermal Units}

New solutions are generated via updating new position for IPSO. The new solutions are then checked and repair for validation.

$P_{i, d}= \begin{cases}P_{i, \max } & \text { if } P_{i, d}>P_{i, \max } \\ P_{i, \min } & \text { if } \quad P_{i, d}<P_{i, \min } ; i=2, \ldots, N \\ P_{i, d} & \text { otherwise }\end{cases}$

\subsection{One System Considering Value Point Effects on Thermal Units}

The search process will be terminate when the current iteration is equal to the maximum number of iterations.

\section{Determining the Best Compromise using Price Penalty Method}

In order to apply the price penalty factor method, the multiobjective function $\mathrm{F}$ in eq. (1) must be reconstructed [21]. When applying the price penalty factor based method for the three dispatch cases, the selection of the three factors are as below.

1) For the economic dispatch, $\Psi_{1}$ is set to 1 and $\Psi_{2}$ is set to 0

2) For the emission dispatch, $\Psi_{1}$ is set to 0 and $\Psi_{2}$ is set to $1 / \mathrm{PR}_{\mathrm{m}}$

3) For the combined economic and emission dispatch, $\Psi_{1}$ and $\Psi_{2}$ are set 1 , and the price penalty factor is calculated by the following steps.

Step 1: Calculate the average fuel cost for each MW of each thermal unit at full generation at each subinterval $\mathrm{m}$.

Step 2: Calculate the average emission for each MW of each thermal unit at full generation at each subinterval $\mathrm{m}$.

Step 3: Calculate the ratio of the average cost to average emission for each thermal unit and thus $\mathrm{PR}_{\mathrm{im}}$ is obtained by

$$
P R_{i m}=\frac{F_{1}\left(P_{s i \max }\right) / P_{s i \max }}{F_{2}\left(P_{s i \max }\right) / P_{s i \max }}(\$ / l b)
$$


Step 4: Arrange the price penalty factor in ascending value order

Step 5: Sum the maximum capacity of each unit $\left(\mathrm{P}_{\mathrm{si}, \max }\right)$ beginning from the full generation of thermal unit with the lowest value of the factor until the sum is equal or higher than the load demand.

Step 6: At this stage, the price penalty factor $\mathrm{PR}_{\mathrm{im}}$ associated with the final unit in the process is chosen as the price penalty factor for the subinterval $\mathrm{m}$.

The values of the factor obtained in [21] have shown that $\mathrm{PR}_{\mathrm{m}}$ depends on the load demand at subinterval $\mathrm{m}$ and note that there is only one value of the factor for each optimal subinterval.

\section{Numerical Results}

The applied IPSO is tested on three systems considering transmission losses consisting of two three unit systems and one ten unit system in which the first two systems neglect valve point loading effects but the last one considers these effects. The proposed algorithm is coded in Matlab platform and run 50 independent trials for each case on a 2.4 $\mathrm{GHz}$ PC with 4 GB of RAM.

\subsection{Study Cases1: Three-unit system considering transmission power losses and with load demand of $850 \mathrm{MW}$}

In this study case 1, three dispatch case including economic dispatch, emission dispatch and combined economic and emission dispatch are considered. The data of the system are from [4]. For implementation of the applied method to the cases, the population size and the maximum number of iterations are respectively set to 10 and 30. In addition, CPSO is also implemented by setting the same values of control parameters for validating the superiority of IPSO. The result from these applied method compared to those from other for the three cases are presented in the Table 1.

Table 1. Comparisons of Result for Case 1

\begin{tabular}{|l|l|l|l|l|l|l|l|}
\hline Dispatch & Method & TS [2] & $\begin{array}{l}\text { NSGA-II } \\
{[4]}\end{array}$ & BBO [7] & CSA [13] & CPSO & IPSO \\
\hline \multirow{2}{*}{ Eco. } & Cost (\$) & 8344.6 & 8344.6 & 8344.59 & 8344.59 & 8344.76 & 8344.59 \\
\cline { 2 - 8 } & Cpu (s) & - & - & - & 0.09 & 0.03 & 0.04 \\
\hline \multirow{3}{*}{ Em. } & Em. (kg) & 0.0958 & 0.09593 & 0.09592 & 0.09592 & 0.09601 & 0.09592 \\
\cline { 2 - 8 } & Cpu (s) & - & - & - & 0.07 & 0.03 & 0.04 \\
\hline \multirow{4}{*}{ CEED } & Cost (\$) & - & 8349.72 & & 8349.722 & 8349.07 & 8349.248 \\
\cline { 2 - 8 } & Em. $(\mathrm{kg})$ & - & 0.09654 & & 0.09654 & 0.10009 & 0.0966 \\
\cline { 2 - 8 } & Cpu (s) & - & - & - & 0.09 & 0.03 & 0.04 \\
\hline
\end{tabular}

It is clear from the table that IPSO is much superior to CPSO for the first two cases, economic dispatch and emission dispatch but there is a trade-off for combined economic and emission dispatch case where IPSO obtains lower cost but higher emission than CPSO. Compared to others, IPSO obtain the same quality of solution as them except Tabu search for the emission dispatch case; however, IPSO is very fast when compared to others, which have been reported execution time. Clearly, the applied improved versions of PSO is very efficient for the study case. 


\subsection{Case 2: Three-unit system considering transmission power losses and with load demand of $400 \mathrm{MW}$}

In the case, a three-unit system supplying to load demand of $400 \mathrm{MW}$ via transmission lines considering power losses is employed. The data of the system are taken from [6]. For implementation of the method to the cases, the population size and the maximum number of iterations are respectively set to 10 and 30 . Table 2 presents the obtained fuel cost and emission from IPSO, and others methods in [6] and [15].

As observed observed from the cost and emission, there is also a trade-off among the applied method and other when comparing both fuel cost and emission. However, the execution time from the applied method is very fast compared to other ones. In conclusion, it is stated that IPSO is a promising method for solving the CEED problem.

Table 2. Comparison of Result for Case 2

\begin{tabular}{|l|l|l|l|l|}
\hline Method & GA [6] & PSO [6] & FPA [15] & IPSO \\
\hline Cost $(\$)$ & 20840.1 & 20838.3 & 20838.1 & 20848.0957 \\
\hline Em.(Kg) & 200.256 & 200.221 & 200.2238 & 200.170 \\
\hline CPU (s) & 0.282 & 0.235 & 0.175 & 0.03 \\
\hline
\end{tabular}

\subsection{Case 3: Ten-unit system considering transmission power losses and with load demand of $2000 \mathrm{MW}$}

In the case, a ten-unit system supplying to load demand of 2000 MW via transmission lines considering power losses is employed. The data of the system are taken from [11]. For implementation of the method to the cases, the population size and the maximum number of iterations are respectively set to 10 and 50. Table 3 presents the obtained fuel cost from IPSO, and others methods, DE [8] and ABC_PSO [11] for economic dispatch. As observed observed from the cost, ABC_PSO and DE obtain the same cost and emission but those from them are higher than that from IPSO by $\$ 2.27$ per hour. Consequently, if the load is run over a day, IPSO will save about $\$ 54.48$ compared to the two methods.

For emission dispatch reported in Table 4, DE has reported the amount of emission of $3923.4 \mathrm{~kg}$; however, we have recalculated the amount and reported in the bracket with $3926.6 \mathrm{~kg}$. It is clear that the proposed IPSO can obtain less emission than ABC_PSO and DE respectively by $5.8396 \mathrm{~kg}$ and $0.1396 \mathrm{~kg}$. for execution time, IPSO is much faster than DE since IPSO has spent only 0.05 second whilst DE has used 9.42 seconds. There was no time reported in [11] fpr ABC_PSO. By giving the evidence of lower emission and faster execution time, it can lead to a conclusion that the proposed IPSO is more effective than ABC_PSO and DE for emission dispatch.

There is also a trade-off among the applied method and other when comparing both fuel cost and emission for the CEED case. In fact, the cost and emission reported in Table 5 have pointed out that the proposed IPSO can obtain better cost but worse emission. However, the execution time from the applied method is very fast compared to other ones. In conclusion, it is stated that IPSO is a promising method for solving the CEED problem. 
Table 3. Comparison for Economic Dispatch of Case 3 with Ten Unit for 2000MW Demand

\begin{tabular}{|l|c|c|c|}
\hline Method & ABC_PSO [11] & DE [8] & \multicolumn{1}{|c|}{ IPSO } \\
\hline Cost $(\$)$ & 111500 & 111500 & $\mathbf{1 1 1 4 9 7 . 7 3}$ \\
\hline Em. $(\mathrm{Kg})$ & 4571.2 & 4581 & 4565.7 \\
\hline Pop. & 15 & 200 & 10 \\
\hline Iterations & 1000 & 100 & 50 \\
\hline Processor & - & $3.0 \mathrm{GHz}$ & 2.4 \\
\hline CPU (s) & - & 9.42 & 0.05 \\
\hline
\end{tabular}

Table 4. Comparison for Emission Dispatch of Case 3 with Ten Unit for 2000MW Demand

\begin{tabular}{|l|c|c|r|}
\hline Method & ABC_PSO [11] & DE [8] & \multicolumn{1}{|c|}{ IPSO } \\
\hline Cost $(\$)$ & 116420 & 116400 & 116410 \\
\hline Em.(Kg) & 3932.3 & $\begin{array}{c}3923.4 \\
(3926.6)\end{array}$ & $\mathbf{3 9 2 6 . 4 6 0 4}$ \\
\hline Pop. & 15 & 200 & 10 \\
\hline Iterations & 1000 & 100 & 50 \\
\hline Processor & - & $3.0 \mathrm{GHz}$ & 2.4 \\
\hline CPU (s) & - & 8.56 & 0.055 \\
\hline
\end{tabular}

Table 5. Comparison for Combined Economic and Emission Dispatch of Case 3 with Ten Units

\begin{tabular}{|l|l|l|l|l|l|l|}
\hline Method & Cost $(\$)$ & Em.(Kg) & Pop. & Iterations & Processor & CPU (s) \\
\hline ABC_PSO [11] & 113420.00 & 4120.10 & 15 & 1000 & & - \\
\hline EMOCA [12] & 113444 & 4113.98 & 50 & 30 & $2 \mathrm{GHz}$ & 2.9 \\
\hline GSA [21] & 113490 & 4111.4 & - & - & - & - \\
\hline FPA [15] & 113370 & 3997.7 & 25 & 500 & & 2.23 \\
\hline MODE [8] & 113480 & 4124.9 & 30 & 50 & $3.0 \mathrm{GHz}$ & 3.82 \\
\hline NSGA-II [8] & 113540 & 4130 & 30 & 50 & $3.0 \mathrm{GHz}$ & 6.02 \\
\hline SPEA 2 [8] & 11352 & 4109.1 & 30 & 20 & $3.0 \mathrm{GHz}$ & 7.53 \\
\hline PDE [8] & 113510 & 4111.4 & 30 & 20 & $3.0 \mathrm{GHz}$ & 4.23 \\
\hline IPSO & 113318.25 & 4132.12 & 10 & 50 & $2.4 \mathrm{GHz}$ & 0.02 \\
\hline
\end{tabular}

The optimal solution including generation, economic, emission and combined economic emission dispatch are given in Table 6 and Table 7 for case 2 and case 3 .

Table 6. Optimal Solution for Case 2 Obtained by the Proposed Method

\begin{tabular}{|c|c|c|c|}
\hline $\begin{array}{c}\mathrm{P}_{\mathrm{i}} \\
(\mathrm{MW})\end{array}$ & $\begin{array}{c}\text { Economic } \\
\text { dispatch }\end{array}$ & $\begin{array}{c}\text { Emission } \\
\text { dispatch }\end{array}$ & $\begin{array}{c}\text { Combined economic } \\
\text { emission dispatch }\end{array}$ \\
\hline 1 & 82.1639 & 105.0889 & 102.5853 \\
\hline 2 & 174.8073 & 151.2134 & 153.7357 \\
\hline 3 & 150.5962 & 151.0955 & 151.0911 \\
\hline
\end{tabular}


Table 7. Optimal Solution for Case 3 Obtained by the Proposed Method

\begin{tabular}{|c|l|l|l|}
\hline $\begin{array}{c}\mathrm{P}_{\mathrm{i}} \\
(\mathrm{MW})\end{array}$ & $\begin{array}{c}\text { Economic } \\
\text { dispatch }\end{array}$ & $\begin{array}{c}\text { Emission } \\
\text { dispatch }\end{array}$ & $\begin{array}{c}\text { Combined economic } \\
\text { emission dispatch }\end{array}$ \\
\hline 1 & 54.9929 & 55.0015 & 53.54744 \\
\hline 2 & 80 & 79.9001 & 76.65635 \\
\hline 3 & 107.3121 & 80.8875 & 84.33669 \\
\hline 4 & 100.202 & 82.247 & 73.32084 \\
\hline 5 & 81.7273 & 160 & 129.7703 \\
\hline 6 & 82.8079 & 240 & 172.7254 \\
\hline 7 & 300 & 294.3100 & 299.555 \\
\hline 8 & 340 & 297.2886 & 309.4935 \\
\hline 9 & 470 & 398.9378 & 454.4212 \\
\hline 10 & 470 & 392.9890 & 430.6503 \\
\hline
\end{tabular}

\section{Conclusion}

In this paper, IPSO has been applied for finding the optimal solutions for CEED problem where both fuel cost, emission and power losses in transmission lines are considered. The IPSO has been constructed by doing several improvements on the conventional method in aim to enhance the optimal solution quality and speed up convergence. The method has been tested on two three-unit systems with transmission losses, convex fuel cost function and emission function and one tenunit system with transmission losses, nonconvex fuel cost function and exponential function. Comparisons between IPSO and CPSO have shown that IPSO is better than CPSO for quality of solutions. In addition, the comparisons among the applied method and other ones have indicated that IPSO is very promising for solving the problem and it will be successfully applied for solving the problems with larger scale and more complex.

\section{References}

[1] D. Kin, M. E. El-Hawary and F. El-Hawary, "Optimal Environmental Dispatching of Electric Power Systems via an Improved Hopfield Neural Network Model”, IEEE Trans. on Power Sysfems, vol. 10, (1995), pp. 1559-1565.

[2] C. A. Roa-Sepulveda, E. R. Salazar-Nova, E. Gracia-Caroca, U.G. Knight and A. Coonick, "Environmental Economic Dispatch via Hopfield Neural Network and Taboo Search", UPEC'96 Universities Power Engineering Conference, Crete, Greece, (1996), pp. 1001-1004.

[3] Y. H. Song, G. S. Wang, P. Y. Wang and A. T. Johns, "Environmental/econoimic dispatch using fuzzy logic controlled genetic algorithm”, IEE Proc-Gener. Transm. Distrib, vol. 44, (1997), pp. 377-382.

[4] C. S. Harry, Rughooputh and Robert T. F. A. King, "Environmental/Economic Dispatch of Thermal Units using an Elitist Multiobjective Evolutionary Algorithm", Industrial Technology, 2003 IEEE International Conference in Maribor, (2003), Slovenia 1, pp. 48-53.

[5] K. K. Mandal and N. Chakraborty, "Effect of Control Parameters on Differential Evolution based Combined Economic Emission Dispatch with Valve-Point Loading and Transmission Loss", International Journal of Emerging Electric Power Systems, vol. 9, (2008), pp. 1-18.

[6] A. L. Devi and O. V. Krishna, "Combined economic and emission dispatch using evolutionary algorithms-a case study", ARPN J Eng Appl Sci., vol. 3, no. 6, (2008), pp. 28-35.

[7] P. K. Roy, S. P. Ghoshal and S. S. Thakur, "Combined economic and emission dispatch problems using biogeography-based optimization”, Electr Eng., vol. 92, (2010), pp. 173-184.

[8] M. Basu, "Economic environmental dispatch using multi-objective differential evolution", Applied Soft Computing, vol. 11, (2011), pp. 2845-2853.

[9] A. Elaiwa, X. Xiab and A. M. Shehata, "Hybrid DE-SQP and hybrid PSO-SQP methods for solving dynamic economic emission dispatch problem with valve-point effects", Electric Power Systems Research, vol. 103, (2013), pp. 192-200.

[10] H. Hamedi, "Solving the combined economic load and emission dispatch problems using new heuristic algorithm", Electrical Power and Energy Systems, vol. 46, (2013), pp. 10-16. 
[11] E. D. Manteaw and N. A Odero, "Combined economic and emission dispatch solution using ABC_PSO hybrid algorithm with valve point loading effect”, International Journal of Sci Res Publ., no. 12, (2012), pp. 1-9.

[12] R. Zhang, J. Zhou, L. Mo, S. Ouyang and X. Liao, "Economic environmental dispatch using an enhanced multi-objective cultural algorithm”, Electr Power Syst Res., vol. 99, (2012), pp. 18-29.

[13] N. T. P. Thao and N. T. Thang, "Environmental Economic Load Dispatch with Quadratic Fuel Cost Function Using Cuckoo Search Algorithm", International Journal of u- and e- Service, Science and Technology, vol. 7, no. 2, (2014), pp. 199-210.

[14] N. T. Thang, "Economic emission load dispatch with multiple fuel options using Hopfiled Lagrange Network", International Journal of Advanced Science and Technology, vol. 57, (2013), pp. 9-24.

[15] A. Abdelaziz, E. Ali and S. A. Elazim, "Implementation of flower pollination algorithm for solving economic load dispatch and combined economic emission dispatch problems in power systems", Energy, vol. 101, (2016), pp 506-518.

[16] L. H. Pham, T. H. Ho, T. T. Nguyen and D. N. Vo, "Modified Bat Algorithm for Combined Economic and Emission Dispatch Problem", Recent Advances in Electrical Engineering and Related Sciences. Lecture Notes in Electrical Engineering, AETA, Springer, Cham, vol. 415, (2016).

[17] U. Guvec, Y. Sonmez, S. Duman and N. Yorukeren, "Combined economic and emission dispatch solution using gravitational search algorithm”, Scientia Iranica., vol. 19, (2012), pp. 1754-1762.

[18] J. Kennedy and R. Eberhart, "Particle swarm optimization", Proceedings of the IEEE Int Conf Neural Networks, (1995), pp. 1942-8.

[19] H. Y. Shi and C. R. Eberhar, "A modified particle swarm optimizer”, IEEE Intl. Conf. on Evolutionary Computation, AK, Anchorage, (1998), pp. 69-73.

[20] C. R. Eberhart and H. Y. Shi, "Comparing inertia weights and constriction factors in particle swarm optimization", Proceedings of the IEEE Congress on Evolutionary Computation, USA, (2000), no. 1, pp. 84-88.

[21] P. S. Kulkarni, A. G. Kothari and D. P. Kothari, "Combined economic and emission dispatch using improved back-propagation neural network”, Electric Power Comp Syst., vol. 28, (2000), pp. 31-44.

\section{Author}

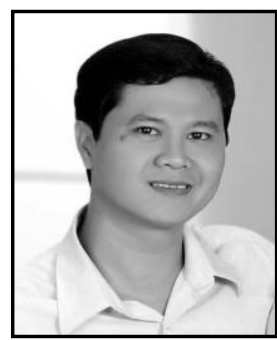

Cuong Duc Minh Nguyen, he received his B.Eng. and M.Eng degrees in Electrical Engineering from University of Technical education Ho Chi Minh City, Ho Chi Minh city, Vietnam in 2008 and 2013, respectively. He is currently teaching at Faculty of Electrical and Electronics Engineering, Ly Tu Trong Technical College, HCM City, Vietnam. His research interests are optimal power flow and improvement of electricity quality. 